\title{
Finite temperature dissipation and transport near quantum critical points
}

\author{
Subir Sachdev \\ Department of Physics, Harvard University, Cambridge MA 02138
}

(Dated: Oct 5, 2009)

\begin{abstract}
I review a variety of model systems and their quantum critical points, motivated by recent experimental and theoretical developments. These are used to present a general discussion of the non-zero temperature crossovers in the vicinity of a quantum critical point. Insights are drawn from the exact solutions of quantum critical transport obtained from the AdS/CFT correspondence. I conclude with a discussion of the role of quantum criticality in the phase diagram of the cuprate superconductors.
\end{abstract}

Contributed chapter to the book 'Developments in Quantum Phase Transitions', edited by Lincoln Carr. 
The author's book ${ }^{\underline{1}}$ on quantum phase transitions has an extensive discussion on the dynamic and transport properties of a variety of systems at non-zero temperatures above a zero temperature quantum critical point. The purpose of the present article is to briefly review some basic material, and to then update the earlier discussion with a focus on experimental and theoretical developments in the decade since the book was written. We note other recent reviews 2 from which portions of this article have been adapted.

We will begin in Section I by introducing a variety of model systems and their quantum critical points; these are motivated by recent experimental and theoretical developments. We will use these systems to introduce basic ideas on the finite temperature crossovers near quantum critical points in Section [I. In Section [II, we will focus on the important quantum critical region and present a general discussion of its transport properties. An important recent development has been the complete exact solution of the dynamic and transport properties in the quantum critical region of a variety of (supersymmetric) model systems in two and higher dimensions: this will be described in Section IV] The exact solutions are found to agree with the earlier general ideas discussed here in Section III. Quite remarkably, the exact solution proceeds via a mapping to the theory of black holes in one higher spatial dimension: we will only briefly mention this mapping here, and refer the reader to the article by Hartnoll in this book for more information. As has often been the case in the history of physics, the existence of a new class of solvable models leads to new and general insights which apply to a much wider class of systems, almost all of which are not exactly solvable. This has also been the case here, as we will review in Section $\mathrm{V}$, a hydrodynamic theory of the low frequency transport properties has been developed, and has led to new relations between a variety of thermo-electric transport co-efficients. Finally, in Section VI we will turn to the cuprate high temperature superconductors, and present recent proposals on how ideas from the theory of quantum phase transition may help unravel the very complex phase diagram of these important materials.

\section{MODEL SYSTEMS AND THEIR CRITICAL THEORIES}

\section{A. Coupled dimer antiferromagnets}

Some of the best studied examples of quantum phase transitions arise in insulators with unpaired $S=1 / 2$ electronic spins residing on the sites, $i$, of a regular lattice. Using $S_{i}^{a}$ $(a=x, y, z)$ to represent the spin $S=1 / 2$ operator on site $i$, the low energy spin excitations are described by the Heisenberg exchange Hamiltonian

$$
H_{J}=\sum_{i<j} J_{i j} S_{i}^{a} \cdot S_{j}^{a}+\ldots
$$




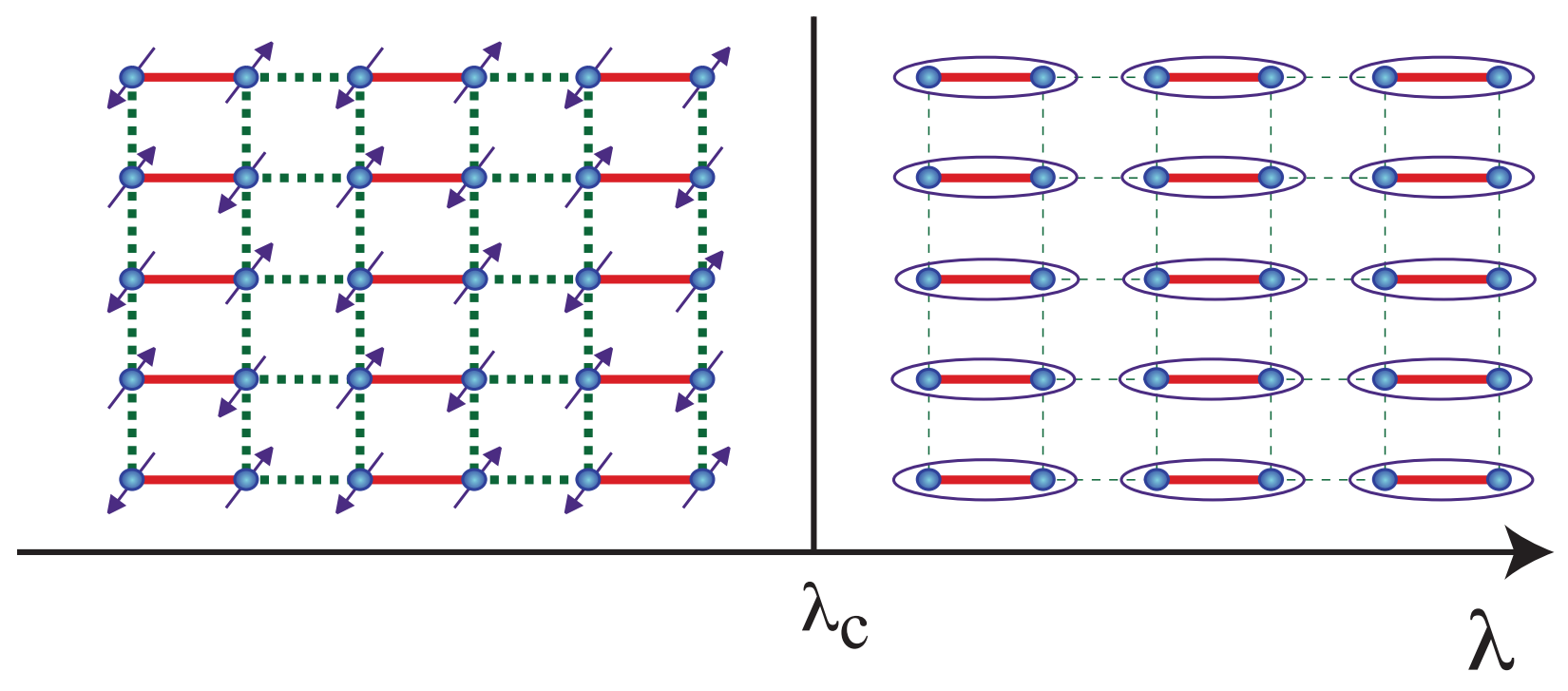

FIG. 1: The coupled dimer antiferromagnet. The full red lines represent an exchange interaction $J$, while the dashed green lines have exchange $J / \lambda$. The ellispes represent a singlet valence bond of spins $(|\uparrow \downarrow\rangle-|\downarrow \uparrow\rangle) / \sqrt{2}$.

where $J_{i j}>0$ is the antiferromagnetic exchange interaction. We will begin with a simple realization of this model is illustrated in Fig. 1. The $S=1 / 2$ spins reside on the sites of a square lattice, and have nearest neighbor exchange equal to either $J$ or $J / \lambda$. Here $\lambda \geq 1$ is a tuning parameter which induces a quantum phase transition in the ground state of this model.

At $\lambda=1$, the model has full square lattice symmetry, and this case is known to have a Néel ground state which breaks spin rotation symmetry. This state has a checkerboard polarization of the spins, just as found in the classical ground state, and as illustrated on the left side of Fig. 1, It can be characterized by a vector order parameter $\varphi^{a}$ which measures the staggered spin polarization

$$
\varphi^{a}=\eta_{i} S_{i}^{a}
$$

where $\eta_{i}= \pm 1$ on the two sublattices of the square lattice. In the Néel state we have $\left\langle\varphi^{a}\right\rangle \neq 0$, and we expect that the low energy excitations can be described by long wavelength fluctuations of a field $\varphi^{a}(x, \tau)$ over space, $x$, and imaginary time $\tau$.

On the other hand, for $\lambda \gg 1$ it is evident from Fig. 1 that the ground state preserves all symmetries of the Hamiltonian: it has total spin $S=0$ and can be considered to be a product of nearest neighbor singlet valence bonds on the $J$ links. It is clear that this state cannot be smoothly connected to the Néel state, and so there must at least one quantum phase transition as a function $\lambda$.

Extensive quantum Monte Carlo simulations ${ }^{3-\underline{5}}$ on this model have shown there is a direct phase transition between these states at a critical $\lambda_{c}$, as in Fig. 11. The value of $\lambda_{c}$ is known accurately, as are the critical exponents characterizing a second-order quantum phase tran- 
sition. These critical exponents are in excellent agreement with the simplest proposal for the critical field theory,$\frac{5}{,}$ which can be obtained via conventional Landau-Ginzburg arguments. Given the vector order parameter $\varphi^{a}$, we write down the action in $d$ spatial and one time dimension,

$$
\mathcal{S}_{L G}=\int d^{d} r d \tau\left[\frac{1}{2}\left[\left(\partial_{\tau} \varphi^{a}\right)^{2}+v^{2}\left(\nabla \varphi^{a}\right)^{2}+s\left(\varphi^{a}\right)^{2}\right]+\frac{u}{4}\left[\left(\varphi^{a}\right)^{2}\right]^{2}\right],
$$

as the simplest action expanded in gradients and powers of $\varphi^{a}$ which is consistent will all the symmetries of the lattice antiferromagnet. The transition is now tuned by varying $s \sim$ $\left(\lambda-\lambda_{c}\right)$. Notice that this model is identical to the Landau-Ginzburg theory for the thermal phase transition in a $d+1$ dimensional ferromagnet, because time appears as just another dimension. As an example of the agreement: the critical exponent of the correlation length, $\nu$, has the same value, $\nu=0.711 \ldots$, to three significant digits in a quantum Monte Carlo study of the coupled dimer antiferromagnet, $\underline{\underline{5}}$ and in a 5 -loop analysis $\underline{6}$ of the renormalization group fixed point of $\mathcal{S}_{L G}$ in $d=2$. Similar excellent agreement is obtained for the doublelayer antiferromagnet $\frac{7 . \underline{\underline{.8}}}{\text { and }}$ the coupled-plaquette antiferromagnet $\underline{\underline{9}}$

In experiments, the best studied realization of the coupled-dimer antiferromagnet is $\mathrm{TlCuCl}_{3}$. In this crystal, the dimers are coupled in all three spatial dimensions, and the transition from the dimerized state to the Néel state can be induced by application of pressure. Neutron scattering experiments by Ruegg and collaborators ${ }^{10}$ have clearly observed the transformation in the excitation spectrum across the transition, as is described by a simple fluctuations analysis about the mean field saddle point of $\mathcal{S}_{L G}$. In the dimerized phase $(s>0)$, a triplet of gapped excitations is observed, corresponding to the three normal modes of $\varphi^{a}$ oscillating about $\varphi^{a}=0$; as expected, this triplet gap vanishes upon approaching the quantum critical point. In a mean field analysis, valid for $d \geq 3$, the field theory in Eq. (1.3) has a triplet gap of $\sqrt{s}$. In the Néel phase, the neutron scattering detects 2 gapless spin waves, and one gapped longitudinal mode $\underline{11}$. This is describe by $\mathcal{S}_{L G}$ for $s<0$, where $\varphi^{a}$ experiences an inverted 'Mexican hat' potential with a minumum at $\left|\varphi^{a}\right|=\sqrt{|s| / v}$. Expanding about this minimum we find that in addition to the gapless spin waves, there is a mode involving amplitude fluctuations of $\left|\varphi^{a}\right|$ which has an energy gap of $\sqrt{2|s|}$. These mean field predictions for the energy of the gapped modes on the two sides of the transition are tested in Fig. 2: the observations are in good agreement with the $1 / 2$ exponent and the predicted $\stackrel{12}{\underline{2}} \sqrt{2}$ ratio, providing a non-trival experimental test of the $\mathcal{S}_{L G}$ field theory.

\section{B. Deconfined criticality}

We now consider an analog of transition discussed in Section IA , but for a Hamiltonian $H=H_{0}+\lambda H_{1}$ which has full square lattice symmetry at all $\lambda$. For $H_{0}$, we choose a form of $H_{J}$, with $J_{i j}=J$ for all nearest neighbor links. Thus at $\lambda=0$ the ground state has Néel 


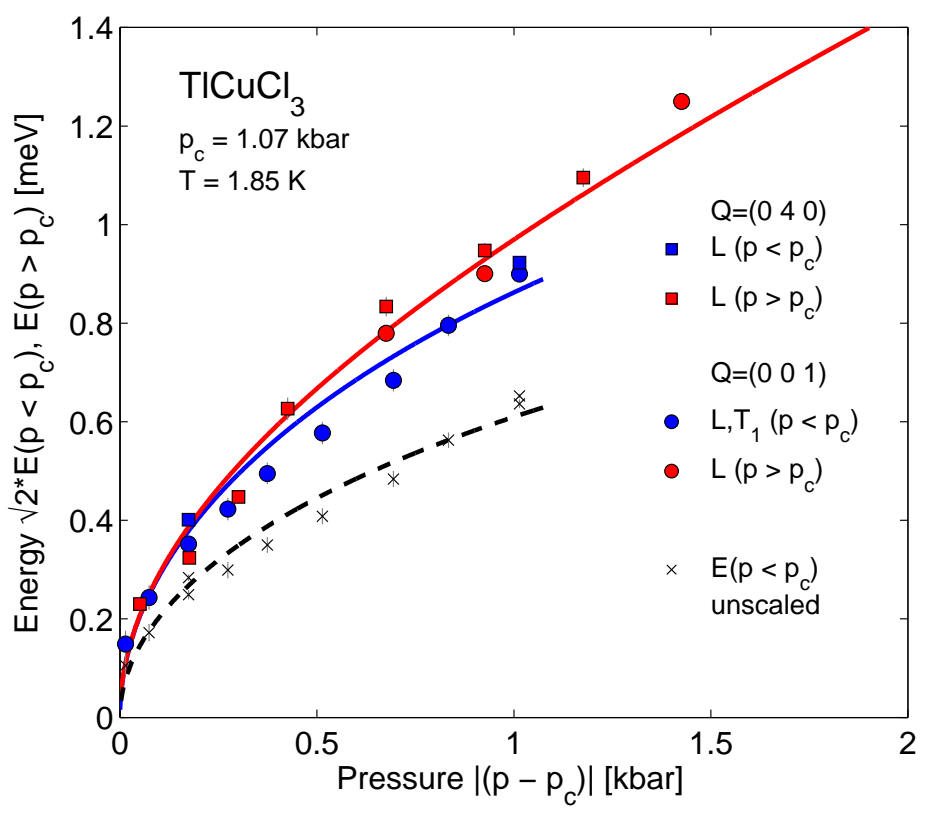

FIG. 2: Energies of the gapped collective modes across the pressure $(p)$ tuned quantum phase

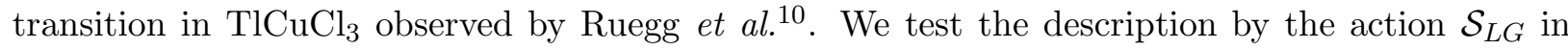
Eq. (1.3) with $s \propto\left(p_{c}-p\right)$ by comparing $\sqrt{2}$ times the energy gap for $p<p_{c}$ with the energy of the longitudinal mode for $p>p_{c}$. The lines are the fits to a $\sqrt{\left|p-p_{c}\right|}$ dependence, testing the $1 / 2$ exponent.

order, as in the left panel of Fig. 1. We now want to choose $H_{1}$ so that increasing $\lambda$ leads to a spin singlet state with spin rotation symmetry restored. A large number of choices have been made in the literature, and the resulting ground state invariably $\underline{13}$ has valence bond solid (VBS) order; a VBS state has been observed in the organic antiferromagnet $\mathrm{EtMe}_{3} \mathrm{P}\left[\mathrm{Pd}(\mathrm{dmit})_{2}\right]_{2} \frac{14,15}{2}$. The VBS state is superficially similar to the dimer singlet state in the right panel of Fig. 1, the spins primarily form valence bonds with near-neighbor sites. However, because of the square lattice symmetry of the Hamiltonian, a columnar arrangement of the valence bonds as in Fig. 1, breaks the square lattice rotation symmetry; there are 4 equivalent columnar states, with the valence bond columns running along different directions. More generally, a VBS state is a spin singlet state, with a non-zero degeneracy due to a spontaneously broken lattice symmetry. Thus a direct transition between the Néel and VBS states involves two distinct broken symmetries: spin rotation symmetry, which is broken only in the Néel state, and a lattice rotation symmetry, which is broken only in the VBS state. The rules of Landau-Ginzburg-Wilson theory imply that there can be no generic second-order transition between such states.

It has been argued that a second-order Néel-VBS transition can indeed occur ${ }^{16}$, but the critical theory is not expressed directly in terms of either order parameter. It involves a fractionalized bosonic spinor $z_{\alpha}(\alpha=\uparrow, \downarrow)$, and an emergent gauge field $A_{\mu}$. The key step is 
to express the vector field $\varphi^{a}$ in terms of $z_{\alpha}$ by

$$
\varphi^{a}=z_{\alpha}^{*} \sigma_{\alpha \beta}^{a} z_{\beta}
$$

where $\sigma^{a}$ are the $2 \times 2$ Pauli matrices. Note that this mapping from $\varphi^{a}$ to $z_{\alpha}$ is redundant. We can make a spacetime-dependent change in the phase of the $z_{\alpha}$ by the field $\theta(x, \tau)$

$$
z_{\alpha} \rightarrow e^{i \theta} z_{\alpha}
$$

and leave $\varphi^{a}$ unchanged. All physical properties must therefore also be invariant under Eq. (1.5), and so the quantum field theory for $z_{\alpha}$ has a U(1) gauge invariance, much like that found in quantum electrodynamics. The effective action for the $z_{\alpha}$ therefore requires introduction of an 'emergent' $\mathrm{U}(1)$ gauge field $A_{\mu}$ (where $\mu=x, \tau$ is a three-component spacetime index). The field $A_{\mu}$ is unrelated the electromagnetic field, but is an internal field which conveniently describes the couplings between the spin excitations of the antiferromagnet. As we did for $\mathcal{S}_{L G}$, we can write down the quantum field theory for $z_{\alpha}$ and $A_{\mu}$ by the constraints of symmetry and gauge invariance, which now yields

$$
\mathcal{S}_{z}=\int d^{2} r d \tau\left[\left|\left(\partial_{\mu}-i A_{\mu}\right) z_{\alpha}\right|^{2}+s\left|z_{\alpha}\right|^{2}+u\left(\left|z_{\alpha}\right|^{2}\right)^{2}+\frac{1}{2 g^{2}}\left(\epsilon_{\mu \nu \lambda} \partial_{\nu} A_{\lambda}\right)^{2}\right]
$$

For brevity, we have now used a "relativistically" invariant notation, and scaled away the spin-wave velocity $v$; the values of the couplings $s, u$ are different from, but related to, those in $\mathcal{S}_{L G}$. The Maxwell action for $A_{\mu}$ is generated from short distance $z_{\alpha}$ fluctuations, and it makes $A_{\mu}$ a dynamical field; its coupling $g$ is unrelated to the electron charge. The action $\mathcal{S}_{z}$ is a valid description of the Néel state for $s<0$ (the critical upper value of $s$ will have fluctuation corrections away from 0), where the gauge theory enters a Higgs phase with $\left\langle z_{\alpha}\right\rangle \neq 0$. This description of the Néel state as a Higgs phase has an analogy with the Weinberg-Salam theory of the weak interactions - in the latter case it is hypothesized that the condensation of a Higgs boson gives a mass to the $W$ and $Z$ gauge bosons, whereas here the condensation of $z_{\alpha}$ quenches the $A_{\mu}$ gauge boson. As written, the $s>0$ phase of $\mathcal{S}_{z}$ is a 'spin liquid' state with a $S=0$ collective gapless excitation associated with the $A_{\mu}$ photon. Non-perturbative effects 13 associated with the monopoles in $A_{\mu}$ (not discussed here), show that this spin liquid is ultimately unstable to the appearance of VBS order.

Numerical studies of the Néel-VBS transition have focussed on a specific lattice antiferromagnet proposed by Sandvik ${ }^{17}-19$. There is strong evidence for VBS order proximate to the Néel state, along with persuasive evidence of a second-order transition. However, some studies $^{20,21}$ support a very weak first order transition. 

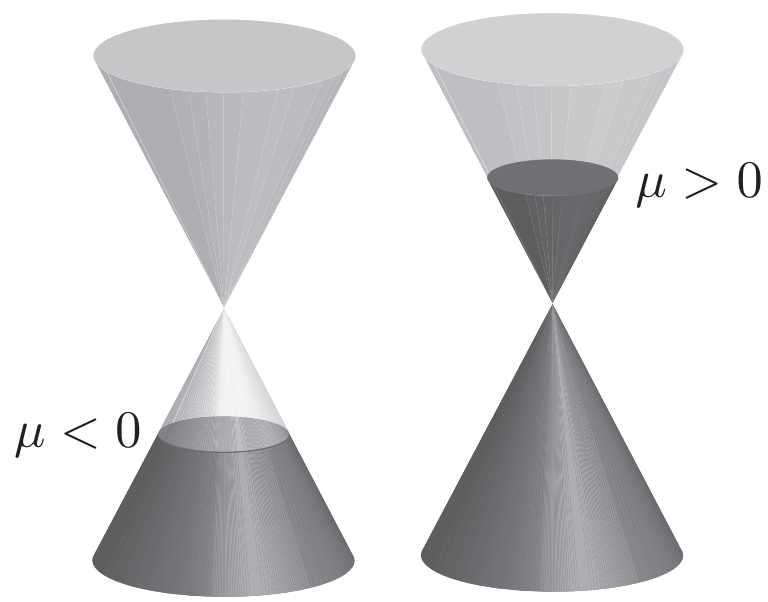

FIG. 3: Dirac dispersion spectrum for graphene showing a 'topological' quantum phase transition from a hole Fermi surface for $\mu<0$ to a electron Fermi surface for $\mu>0$.

\section{Graphene}

The last few years have seen an explosion in experimental and theoretical studies 22 of graphene: a single hexagonal layer of carbon atoms. At the currently observed temperatures, there is no evident broken symmetry in the electronic excitations, and so it is not conventional to think of graphene as being in the vicinity of a quantum critical point. However, graphene does indeed undergo a bona fide quantum phase transition, but one without any order parameters or broken symmetry. This transition may be viewed as being 'topological' in character, and is associated with a change in nature of the Fermi surface as a function of carrier density.

Pure, undoped graphene has a conical electronic dispersion spectrum at two points in the Brillouin zone, with the Fermi energy at the particle-hole symmetric point at the apex of the cone. So there is no Fermi surface, just a Fermi point, where the electronic energy vanishes, and pure graphene is a 'semi-metal'. By applying a bias voltage, the Fermi energy can move away from this symmetric point, and a circular Fermi surface develops, as illustrated in Fig. 3. The Fermi surface is electron-like for one sign of the bias, and hole-like for the other sign. This change from electron to hole character as a function of bias voltage constitutes the quantum phase transition in graphene. As we will see below, with regard to its dynamic properties near zero bias, graphene behaves in almost all respects like a canonical quantum critical system.

The field theory for graphene involves fermionic degrees of freedom. Representing the electronic orbitals near one of the Dirac points by the two-component fermionic spinor $\Psi_{a}$, where $a$ is a sublattice index (we suppress spin and 'valley' indices), we have the effective 
electronic action

$$
\begin{gathered}
\mathcal{S}_{\Psi}=\int d^{2} r \int d \tau \Psi_{a}^{\dagger}\left[\left(\partial_{\tau}+i A_{\tau}-\mu\right) \delta_{a b}+i v_{F} \tau_{a b}^{x} \partial_{x}+i v_{F} \tau_{a b}^{y} \partial_{y}\right] \Psi_{b} \\
+\frac{1}{2 g^{2}} \int \frac{d^{2} q}{4 \pi^{2}} \int d \tau \frac{q}{2 \pi}\left|A_{\tau}(\mathbf{q}, \tau)\right|^{2}
\end{gathered}
$$

where $\tau_{a b}^{i}$ are Pauli matrices in the sublattice space, $\mu$ is the chemical potential, $v_{F}$ is the Fermi velocity, and $A_{\tau}$ is the scalar potential mediating the Coulomb interaction with coupling $g^{2}=e^{2} / \epsilon$ ( $\epsilon$ is a dielectric constant). This theory undergoes a quantum phase transition as a function of $\mu$, at $\mu=0$, similar in many ways to that of $\mathcal{S}_{L G}$ as a function of $s$. The interaction between the fermionic excitations here has coupling $g^{2}$, which is the analog of the non-linearity $u$ in $\mathcal{S}_{L G}$. However, while $u$ scaled to a fixed point non-zero fixed point value under the renormalization group flow, $g$ flows logarithmically slowly to zero. For many purposes, it is safe to ignore this flow, and to set $g$ equal to fixed value; this value is characterized by the dimensionless 'fine structure constant' $\alpha=g^{2} /\left(\hbar v_{F}\right)$ which is of order unity in graphene.

\section{Spin density waves}

Finally, we consider the onset of Néel order, as in Section IA. but in a metal rather than an insulator. It is conventional to refer to such metallic Néel states as have spin density wave (SDW) order. Our discussion here is motivated by application to the cuprate superconductors: there is good evidence 23,24 that the transition we describe below is present in the electron-doped cuprates, and proposals of its application to the hole-doped cuprates will be discussed in Section VI.

We begin with the band structure describing the cuprates in the over-doped region, well away from the Mott insulator. Here the electrons $c_{i \alpha}$ are described by the Hamiltonian

$$
H_{c}=-\sum_{i<j} t_{i j} c_{i \alpha}^{\dagger} c_{i \alpha} \equiv \sum_{\mathbf{k}} \varepsilon_{\mathbf{k}} c_{\mathbf{k} \alpha}^{\dagger} c_{\mathbf{k} \alpha}
$$

with $t_{i j}$ non-zero for first, second and third neighbors on the square lattice. This leads to Fermi surface shown in the right-most panel of Fig. 4. As in Section [C], we will consider topological changes to this Fermi surface, but is induced here by a conventional SDW (Néel) order parameter $\varphi^{a}$. From the structure of the ordering in Fig. 1 we see that $\varphi^{a}$ carries

momentum $\mathbf{K}=(\pi, \pi)$, and so will transfer momentum $\mathbf{K}$ while scattering the $c_{\alpha}$ fermions; this leads to the coupling

$$
H_{\mathrm{sdw}}=\varphi^{a} \sum_{\mathbf{k}, \alpha, \beta} c_{\mathbf{k}, \alpha}^{\dagger} \sigma_{\alpha \beta}^{a} c_{\mathbf{k}+\mathbf{K}, \beta}
$$

We can now follow the evolution of the Fermi surface under the onset of SDW order by 


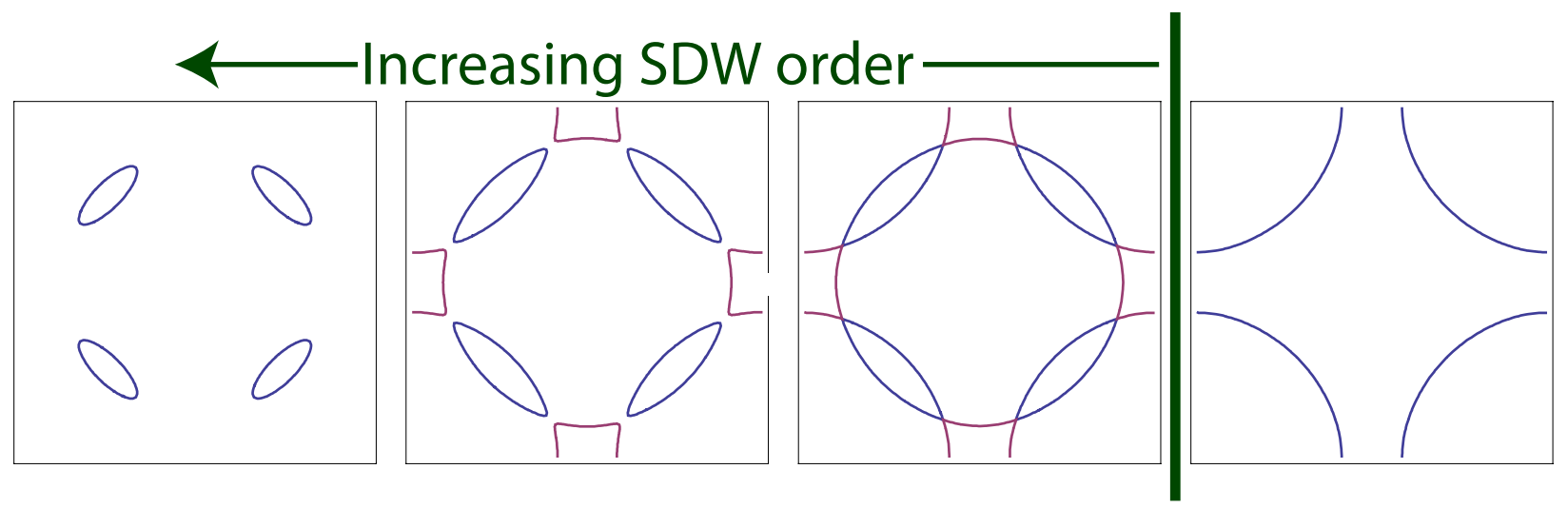

FIG. 4: Evolution of the Fermi surface of the hole doped cuprates in a conventional SDW theory 25 as a function of the magnitude of the SDW order $\left|\varphi^{a}\right|$. The right panel is the large Fermi surface state with no SDW order, with states contiguous to $\mathbf{k}=0$ occupied by electrons. The onset of SDW order induces the formation of electron (red) and hole (blue) pockets. With further increase of $\left|\varphi^{a}\right|$, the electron pockets disappear and only hole pockets remain (the converse happens in the last step for the electron-doped cuprates.

diagonalizing $H_{c}+H_{\mathrm{sdw}}$ for constant $\varphi^{a}$ : the results of this ${ }^{25}$ are shown in Fig. 4. The second panel from the right shows the Fermi surface obtained by translating the original Fermi surface by $\mathbf{K}$, and the remaining panels show the consequences of mixing between the states at momentum $\mathbf{k}$ and $\mathbf{k}+\mathbf{K}$.

We are now interested in the nature of the quantum critical point at the onset of SDW order, indicated by the vertical line in Fig. 4. Note that this transition combines the features of Sections $\amalg \mathrm{A}$ and $\amalg \mathrm{C}$; it has an order parameter, as in the coupled dimer antiferromagnet, and it has a topological change in the Fermi surface, as in graphene. Thus a complete theory is just the combination of these two features: $\mathcal{S}_{L G}+H_{c}+H_{\mathrm{sdw}}$. A common assertion 26,27 is that we can pay less attention to the Fermi surface change by simply integrating out the $c_{\alpha}$ fermions and working with the resulting modified action for $\varphi^{a}$. Right at the quantum critical point, the SDW fluctuations, $\varphi^{a}$ connect points on the large Fermi surface, and so can decay into a large density of states of particle-hole excitations. The damping induced by this particle-hole continuum modifies the effective action for $\varphi^{a}$ from Eq. (1.3) by adding a relevant dissipative term: 26,27

$$
\mathcal{S}_{H}=\mathcal{S}_{L G}+\int \frac{d^{2} k}{4 \pi^{2}} \int \frac{d \omega}{2 \pi}|\omega|\left|\varphi^{a}(k, \omega)\right|^{2}
$$

More recent analyses ${ }^{28}$ have indicated that this procedure is likely not correct in two spatial dimensions, and that the Fermi surface change has to be treated in a more fundamental manner. 


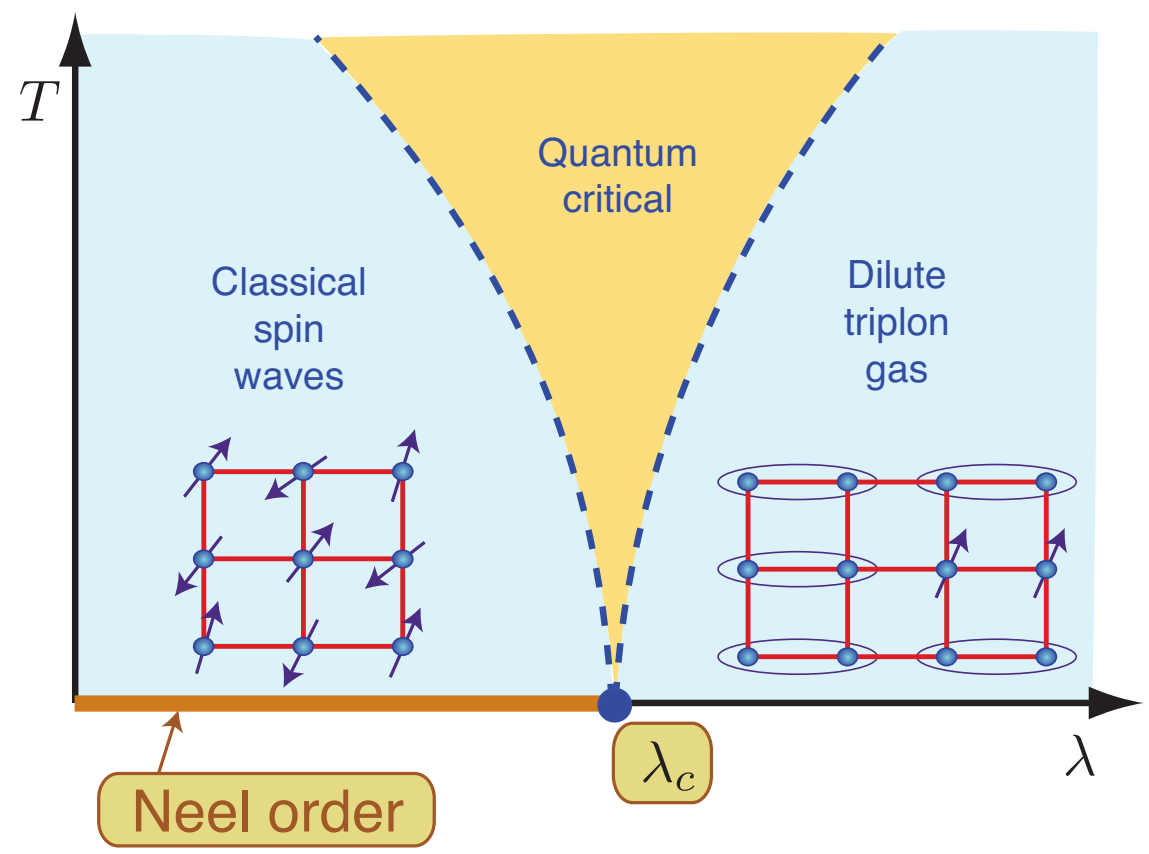

FIG. 5: Finite temperature crossovers of the coupled dimer antiferromagnet in Fig. 1 ,

\section{FINITE TEMPERATURE CROSSOVERS}

The previous section has described four model systems at $T=0$ : we examined the change in the nature of the ground state as a function of some tuning parameter, and motivated a quantum field theory which describes the low energy excitations on both sides of the quantum critical point.

We now turn to the important question of the physics at non-zero temperatures. All of the models share some common features, which we will first explore for the coupled dimer antiferromagnet. For $\lambda>\lambda_{c}$ (or $s>0$ in $\mathcal{S}_{L G}$ ), the excitations consist of a triplet of $S=1$ particles (the 'triplons'), which can be understood perturbatively in the large $\lambda$ expansion as an excited $S=1$ state on a dimer, hopping between dimers (see Fig. (5). The mean field theory tells us that the excitation energy of this dimer vanishes as $\sqrt{s}$ upon approaching the quantum critical point. Fluctuations beyond mean field, described by $\mathcal{S}_{L G}$, show that the exponent is modified to $s^{z \nu}$, where $z=1$ is the dynamic critical exponent, and $\nu$ is the correlation length exponent. Now imagine turning on a non-zero temperature. As long as $T$ is smaller than the triplon gap, i.e. $T<s^{z \nu}$, we expect a description in terms of a dilute gas of thermally excited triplon particles. This leads to the behavior shown on the right-hand-side of Fig. 5, delimited by the crossover indicted by the dashed line. Note that the crossover line approaches $T=0$ only at the quantum critical point.

Now let us look a the complementary behavior at $T>0$ on the Néel-ordered side of the transition, with $s<0$. In two spatial dimensions, thermal fluctuations prohibit the breaking of a non-Abelian symmetry at all $T>0$, and so spin rotation symmetry is immediately 
restored. Nevertheless, there is an exponentially large spin correlation length, $\xi$, and at distances shorter than $\xi$ we can use the ordered ground state to understand the nature of the excitations. Along with the spin-waves, we also found the longitudinal 'Higgs' mode with energy $\sqrt{-2 s}$ in mean field theory. Thus, just as was this case for $s>0$, we expect this spin-wave+Higgs picture to apply at all temperatures lower than the natural energy scale; i.e. for $T<(-s)^{z \nu}$. This leads to the crossover boundary shown on the left-hand-side of Fig. 5 .

Having delineated the physics on the two sides of the transition, we are left with the crucial quantum critical region in the center of Fig. 5. This is present for $T>|s|^{z \nu}$, i.e. at higher temperatures in the vicinity of the quantum critical point. To the left of the quantum critical region, we have a description of the dynamics and transport in terms of an effectively classical model of spin waves: this is the 'renormalized classical' regime of Ref. 29 . To the right of the quantum critical region, we again have a regime of classical dynamics, but now in terms of a Boltzmann equation for the triplon particles. A key property of quantum critical region is that there is no description in terms of either classical particles or classical waves at the times of order the typical relaxation time, $\tau_{r}$, of thermal excitations. Instead, quantum and thermal effects are equally important, and involve the non-trivial dynamics of the fixedpoint theory describing the quantum critical point. Note that while the fixed-point theory applies only at a single point $\left(\lambda=\lambda_{c}\right)$ at $T=0$, its influence broadens into the quantum critical region at $T>0$. Because there is no characteristing energy scale associated with the fixed-point theory, $k_{B} T$ is the only energy scale available to determine $\tau_{r}$ at non-zero temperatures. Thus, in the quantum critical region

$$
\tau_{r}=\mathcal{C} \frac{\hbar}{k_{B} T}
$$

where $\mathcal{C}$ is a universal constant dependent only upon the universality class of the fixed point theory i.e. it is universal number just like the critical exponents. This value of $\tau_{r}$ determines the 'friction coefficients' associated with the dissipative relaxation of spin fluctuations in the quantum critical region. It is also important for the transport co-efficients associated with conserved quantities, and this will be discussed in Section III.

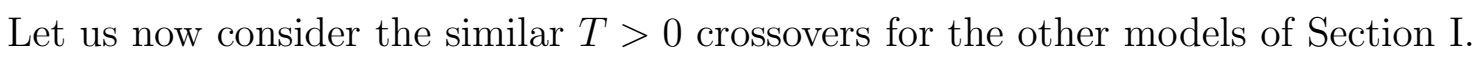

The Néel-VBS transition of Section IB has crossovers very similar to those in Fig. 5 , with one important difference. The VBS state breaks a discrete lattice symmetry, and this symmetry remains broken for a finite range of non-zero temperatures. Thus, within the right-hand 'triplon gas' regime of Fig. 5, there is a phase transition line at a critical temperature $T_{\mathrm{VBS}}$. The value of $T_{\mathrm{VBS}}$ vanishes very rapidly as $s \searrow 0$, and is controlled by the non-perturbative monopole effects which were briefly noted in Section IB.

For graphene, the discussion above applied to Fig. 3 leads to the crossover diagram shown in Fig. 6, as noted by Sheehy and Schmalian ${ }^{31}$. We have the Fermi liquid regimes of the 


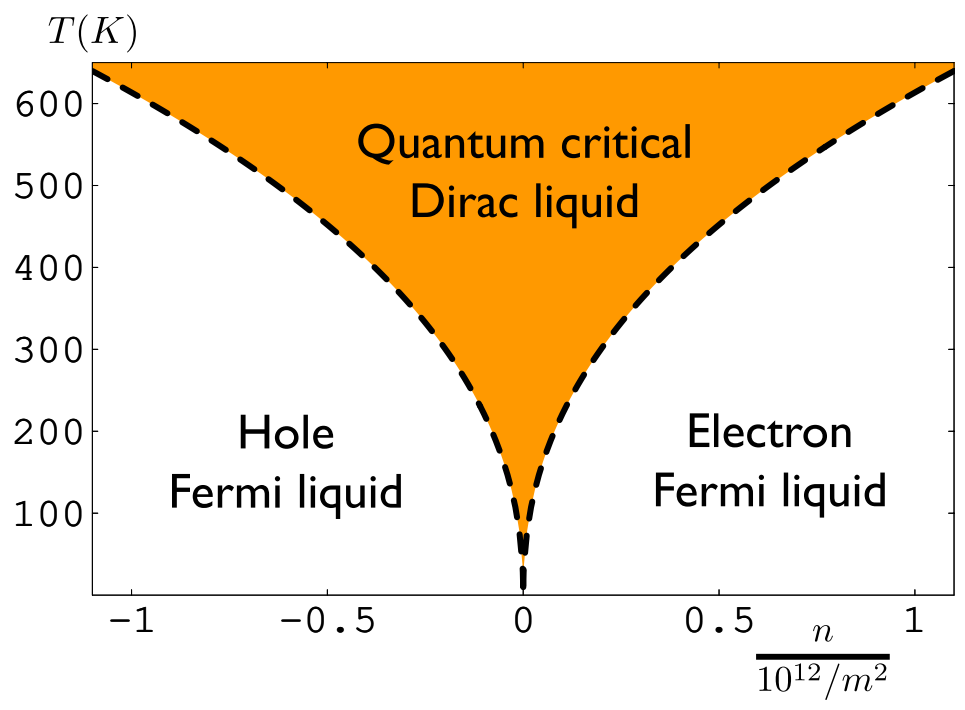

FIG. 6: Finite temperature crossovers of graphene as a function of electron density $n$ (which is tuned by $\mu$ in Eq. (1.7)) and temperature, T. Adapted from Ref. 31 .

electron- and hole-like Fermi surfaces on either side of the critical point, along with an intermediate quantum critical Dirac liquid. A new feature here is related to the logarithmic flow of the dimensionless 'fine structure constant' $\alpha$ controlling the Coulomb interactions, which was noted in Section IC. In the quantum critical region, this constant takes the typical value $\alpha \sim 1 / \ln (1 / T)$. Consequently for the relaxation time in Eq. (2.1) we have $\mathcal{C} \sim \ln ^{2}(1 / T)$. This time determines both the width of the electron spectral functions, and also the transport co-efficients, as we will see in Section III.

Finally, we turn to the spin density wave transition of Section ID. From the evolution in Fig. 4 and the discussion above, we have the crossover phase diagram ${ }^{32}$ in Fig. 7 , As in Fig. 5, there is no transition at non-zero temperatures, and now the crossover is between the topologically distinct Fermi surface configurations of Fig. 4. The Hertz action for the SDW fluctuations in Eq. (1.8) predicts ${ }^{28}, 32$ logarithmic corrections to the leading scaling behavior, similar to those for graphene. The interplay of such SDW fluctuations with the topological change in the Fermi surface configuration is not fully understood ${ }^{33}$, and is labeled as the 'strange metal' regime in Fig. 7 ,

\section{QUANTUM CRITICAL TRANSPORT}

We now turn to the 'transport' properties in the quantum critical region: we consider the response functions associated with any globally conserved quantity. For the antiferromagnetic systems in Sections $\llbracket \mathrm{A}$ and $[\mathrm{IB}$, this requires consideration of the transport of total spin, and the associated spin conductivities and diffusivities. For graphene, we can consider charge and momentum transport. Our discussion below will also apply to the superfluidinsulator transition: for bosons in a periodic potential, this transition is described ${ }^{34}$ by a field 


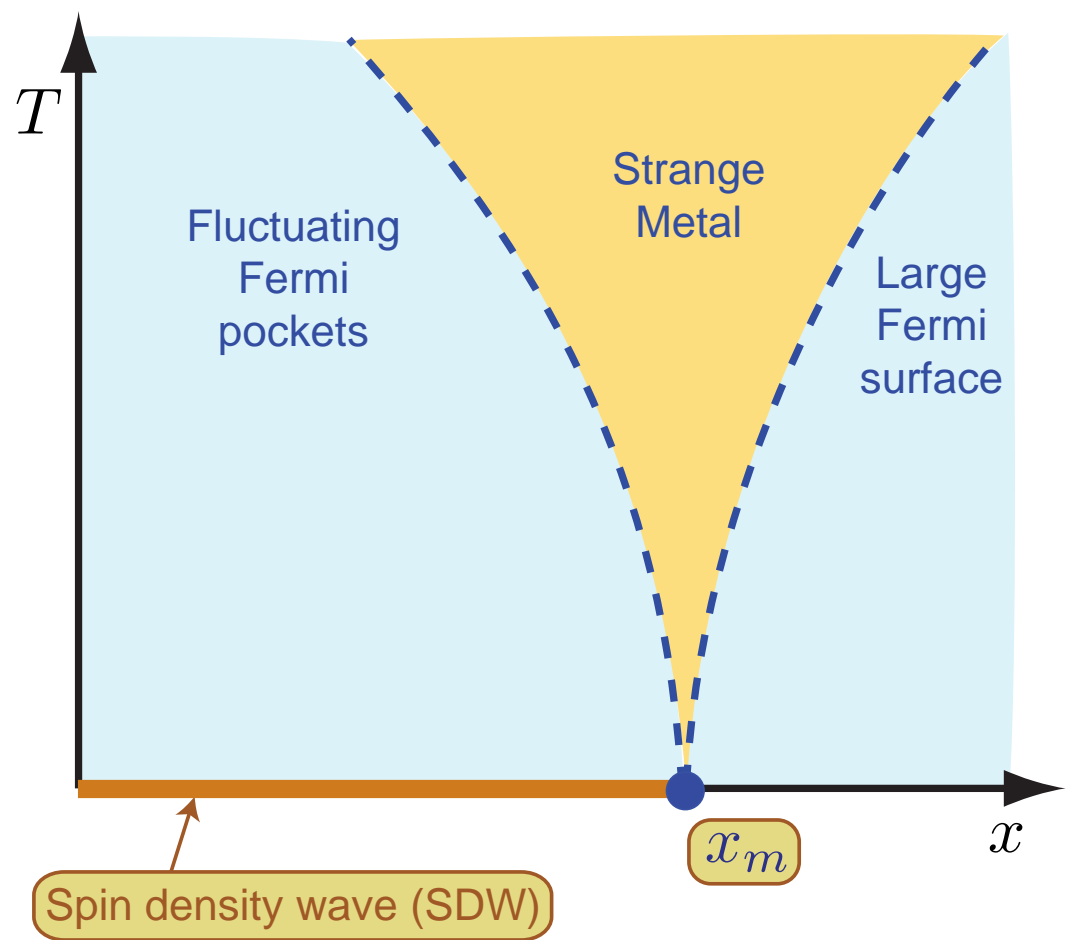

FIG. 7: Finite temperature crossovers of near the SDW ordering transition of Fig. 4, Here, looking to application to the cuprates to be discussed in Section VI, we have assumed that the carrier density, $x$, tunes the system across the SDW transition.

theory closely related to that in Eq. (1.3). However, we will primarily use a language appropriate to charge transport in graphene below. We will describe the properties of a generic strongly-coupled quantum critical point and mention, where appropriate, the changes due to the logarithmic flow of the coupling in graphene.

In traditional condensed matter physics, transport is described by identifying the lowlying excitations of the quantum ground state, and writing down 'transport equations' for the conserved charges carried by them. Often, these excitations have a particle-like nature, such as the 'triplon' particles of Fig. 5 or the electron or hole quasiparticles of the Fermi liquids in Fig. 6. In other cases, the low-lying excitations are waves, such as the spinwaves in Fig. 5, and their transport is described by a non-linear wave equation (such as the Gross-Pitaevski equation). However, as we have discussed in Section [I neither description is possible in the quantum critical region, because the excitations do not have a particle-like or wave-like character.

Despite the absence of an intuitive description of the quantum critical dynamics, we can expect that the transport properties should have a universal character determined by the quantum field theory of the quantum critical point. In addition to describing single excitations, this field theory also determines the $S$-matrix of these excitations by the renormalization group fixed-point value of the couplings, and these should be sufficient to determine transport properties 35 . The transport co-efficients, and the relaxation time to local equilib- 
rium, are not proportional to a mean free scattering time between the excitations, as is the case in the Boltzmann theory of quasiparticles. Such a time would typically depend upon the interaction strength between the particles. Rather, the system behaves like a "perfect fluid" in which the relaxation time is as short as possible, and is determined universally by the absolute temperature, as indicated in Eq. (2.1). Indeed, it was conjectured in Ref. 1 that the relaxation time in Eq. (2.1) is a generic lower bound for interacting quantum systems. Thus the non-quantum-critical regimes of all the phase diagrams in Section II have relaxation times which are all longer than Eq. (2.1).

The transport co-efficients of this quantum-critical perfect fluid also do not depend upon the interaction strength, and can be connected to the fundamental constants of nature. In particular, the electrical conductivity, $\sigma$, is given by (in two spatial dimensions) $)^{35}$

$$
\sigma_{Q}=\frac{e^{* 2}}{h} \Phi_{\sigma},
$$

where $\Phi_{\sigma}$ is a universal dimensionless constant of order unity, and we have added the subscript $Q$ to emphasize that this is the conductivity for the case of graphene with the Fermi level at the Dirac point (for the superfluid-insulator transition, this would correspond to bosons at integer filling) with no impurity scattering, and at zero magnetic field. Here $e^{*}$ is the charge of the carriers: for a superfluid-insulator transition of Cooper pairs, we have $e^{*}=2 e$, while for graphene we have $e^{*}=e$. The renormalization group flow of the 'fine structure constant' $\alpha$ of graphene to zero at asymptotically low $T$, allows an exact computation in this case $\mathrm{e}^{36}: \Phi_{\sigma} \approx 0.05 \ln ^{2}(1 / T)$. For the superfluid-insulator transition, $\Phi_{\sigma}$ is $T$-independent (this is the generic situation with non-zero fixed point values of the interaction ${ }^{37}$ ) but it has only been computed 1.35 to leading order in expansions in $1 / N$ (where $N$ is the number of order parameter components) and in $3-d$ (where $d$ is the spatial dimensionality). However, both expansions are neither straightforward nor rigorous, and require a physically motivated resummation of the bare perturbative expansion to all orders. It would therefore be valuable to have exact solutions of quantum critical transport where the above results can be tested, and we turn to such solutions in the next section.

In addition to charge transport, we can also consider momentum transport. This was considered in the context of applications to the quark-gluon plasma $\stackrel{38}{ }$; application of the analysis of Ref. $\frac{35}{5}$ shows that the viscosity, $\eta$, is given by

$$
\frac{\eta}{s}=\frac{\hbar}{k_{B}} \Phi_{\eta},
$$

where $s$ is the entropy density, and again $\Phi_{\eta}$ is a universal constant of order unity. The value of $\Phi_{\eta}$ has recently been computed $\underline{\underline{41}}$ for graphene, and again has a logarithmic $T$ dependence because of the marginally irrelevant interaction: $\Phi_{\eta} \approx 0.008 \ln ^{2}(1 / T)$.

We conclude this section by discussing some subtle aspects of the physics behind the 
seemingly simple result quantum-critical in Eq. (3.1). For simplicity, we will consider the case of a "relativistically" invariant quantum critical point in $2+1$ dimensions (such as the field theories of Section 【A and [B, but marginally violated by graphene, a subtlety we ignore below). Consider the retarded correlation function of the charge density, $\chi(k, \omega)$, where $k=|\mathbf{k}|$ is the wavevector, and $\omega$ is frequency; the dynamic conductivity, $\sigma(\omega)$, is related to $\chi$ by the Kubo formula,

$$
\sigma(\omega)=\lim _{k \rightarrow 0} \frac{-i \omega}{k^{2}} \chi(k, \omega) .
$$

It was argued in Ref. $\underline{\underline{35}}$ that despite the absence of particle-like excitations of the critical ground state, the central characteristic of the transport is a crossover from collisionless to collision-dominated transport. At high frequencies or low temperatures, the limiting form for $\chi$ reduces to that at $T=0$, which is completely determined by relativistic and scale invariance and current conversion upto an overall constant

$$
\chi(k, \omega)=\frac{e^{* 2}}{h} K \frac{k^{2}}{\sqrt{v^{2} k^{2}-(\omega+i \eta)^{2}}} \quad, \quad \sigma(\omega)=\frac{e^{* 2}}{h} K \quad ; \quad \hbar \omega \gg k_{B} T,
$$

where $K$ is a universal number $\underline{\underline{39}}$. However, phase-randomizing collisions are intrinsically present in any strongly interacting critical point (above one spatial dimension) and these lead to relaxation of perturbations to local equilibrium and the consequent emergence of hydrodynamic behavior. So at low frequencies, we have instead an Einstein relation which determines the conductivity with

$$
\chi(k, \omega)=e^{* 2} \chi_{c} \frac{D k^{2}}{D k^{2}-i \omega}, \quad \sigma(\omega)=e^{* 2} \chi_{c} D=\frac{e^{* 2}}{h} \Theta_{1} \Theta_{2} ; \quad \hbar \omega \ll k_{B} T,
$$

where $\chi_{c}$ is the compressibility and $D$ is the charge diffusion constant. Quantum critical scaling arguments show that the latter quantities obey

$$
\chi_{c}=\Theta_{1} \frac{k_{B} T}{h^{2} v^{2}}, \quad D=\Theta_{2} \frac{h v^{2}}{k_{B} T},
$$

where $\Theta_{1,2}$ are universal numbers. A large number of papers in the literature, particularly those on critical points in quantum Hall systems, have used the collisionless method of Eq. (3.4) to compute the conductivity. However, the correct d.c. limit is given by Eq. (3.5), and the universal constant in Eq. (3.1) is given by $\Phi_{\sigma}=\Theta_{1} \Theta_{2}$. Given the distinct physical interpretation of the collisionless and collision-dominated regimes, we expect that $K \neq \Theta_{1} \Theta_{2}$. This has been shown in a resummed perturbation expansion for a number of quantum critical points 1 . 


\section{EXACT RESULTS FOR QUANTUM CRITICAL TRANSPORT}

The results of Section [II] were obtained by using physical arguments to motivate resummations of perturbative expansions. Here we shall support the ad hoc assumptions behind these results by examining an exactly solvable model of quantum critical transport.

The solvable model may be viewed as a generalization of the gauge theory in Eq. (1.6) to the maximal possible supersymmetry. In $2+1$ dimensions, this is known as $\mathcal{N}=8$ supersymmetry. Such a theory with the U(1) gauge group is free, and so we consider the non-Abelian Yang-Millis theory with a $\mathrm{SU}(N)$ gauge group. The resulting supersymmetric Yang-Mills (SYM) theory has only one coupling constant, which is the analog of the electric charge $g$ in Eq. (1.6). The matter content is naturally more complicated than the complex scalar $z_{\alpha}$ in Eq. (1.6), and also involves relativistic Dirac fermions as in Eq. (1.7). However all the terms in the action for the matter fields are also uniquely fixed by the single coupling constant $g$. Under the renormalization group, it is believed that $g$ flows to an attractive fixed point at a non-zero coupling $g=g^{*}$; the fixed point then defines a supersymmetric conformal field theory in $2+1$ dimensions (a SCFT3), and we are interested here in the transport properties of this SCFT3.

A remarkable recent advance has been the exact solution of this SCFT3 in the $N \rightarrow \infty$ limit using the AdS/CFT correspondence ${ }^{42}$. The solution proceeds by a dual formulation as a four-dimensional supergravity theory on a spacetime with uniform negative curvature: anti-de Sitter space, or $\mathrm{AdS}_{4}$. Remarkably, the solution is also easily extended to non-zero temperatures, and allows direct computation of the correlators of conserved charges in real time. At $T>0$ a black hole appears in the gravity, resulting in an AdS-Schwarzschild spacetime, and $T$ is also the Hawking temperature of the black hole; the real time solutions also extend to $T>0$.

The results of a full computation 43 of the density correlation function, $\chi(k, \omega)$ are shown in Fig. 8 and 9. The most important feature of these results is that the expected limiting forms in the collisionless (Eq. (3.4) ) and collision-dominated (Eq. (3.5) are obeyed. Thus the results do display the collisionless to collision-dominated crossover at a frequency of order $k_{B} T / \hbar$, as was postulated in Section III.

An additional important feature of the solution is apparent upon describing the full structure of both the density and current correlations. Using spacetime indices $(\mu, \nu=t, x, y)$ we can represent these as the tensor $\chi_{\mu \nu}(\mathbf{k}, \omega)$, where the previously considered $\chi \equiv \chi_{t t}$. At $T>0$, we do not expect $\chi_{\mu \nu}$ to be relativistically covariant, and so can only constrain it by spatial isotropy and density conservation. Introducing a spacetime momentum $p_{\mu}=(\omega, \mathbf{k})$, and setting the velocity $v=1$, these two constraints lead to the most general form

$$
\chi_{\mu \nu}(\mathbf{k}, \omega)=\frac{e^{* 2}}{h} \sqrt{p^{2}}\left(P_{\mu \nu}^{T} K^{T}(k, \omega)+P_{\mu \nu}^{L} K^{L}(k, \omega)\right)
$$

where $p^{2}=\eta^{\mu \nu} p_{\mu} p_{\nu}$ with $\eta_{\mu \nu}=\operatorname{diag}(-1,1,1)$, and $P_{\mu \nu}^{T}$ and $P_{\mu \nu}^{L}$ are orthogonal projectors 


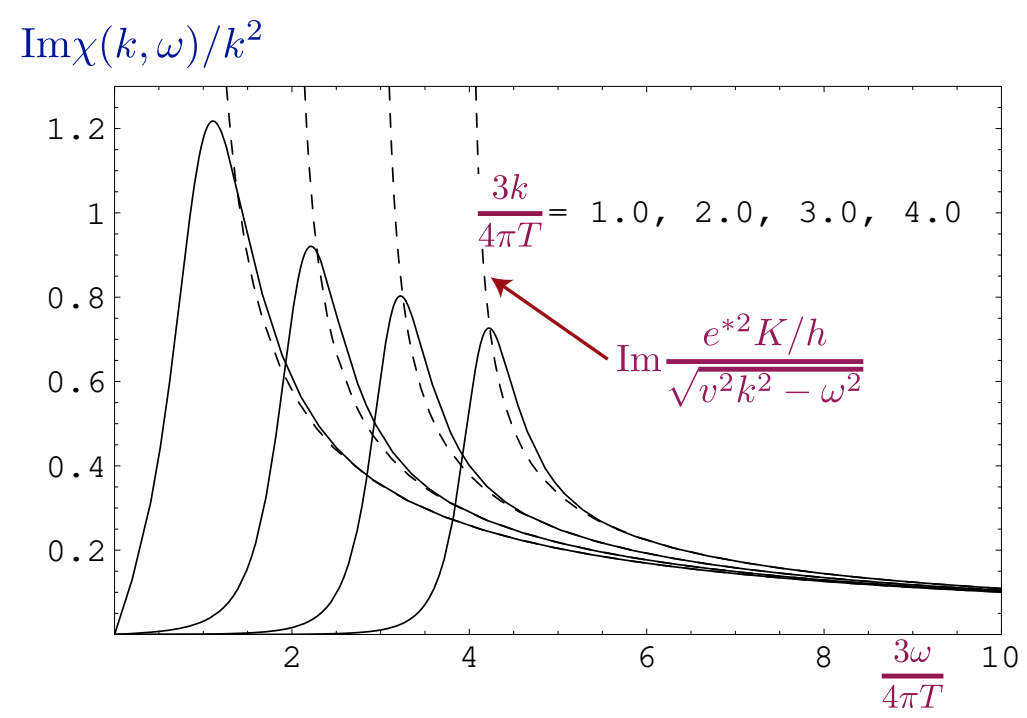

FIG. 8: Spectral weight of the density correlation function of the SCFT3 with $\mathcal{N}=8$ supersymmetry in the collisionless regime.

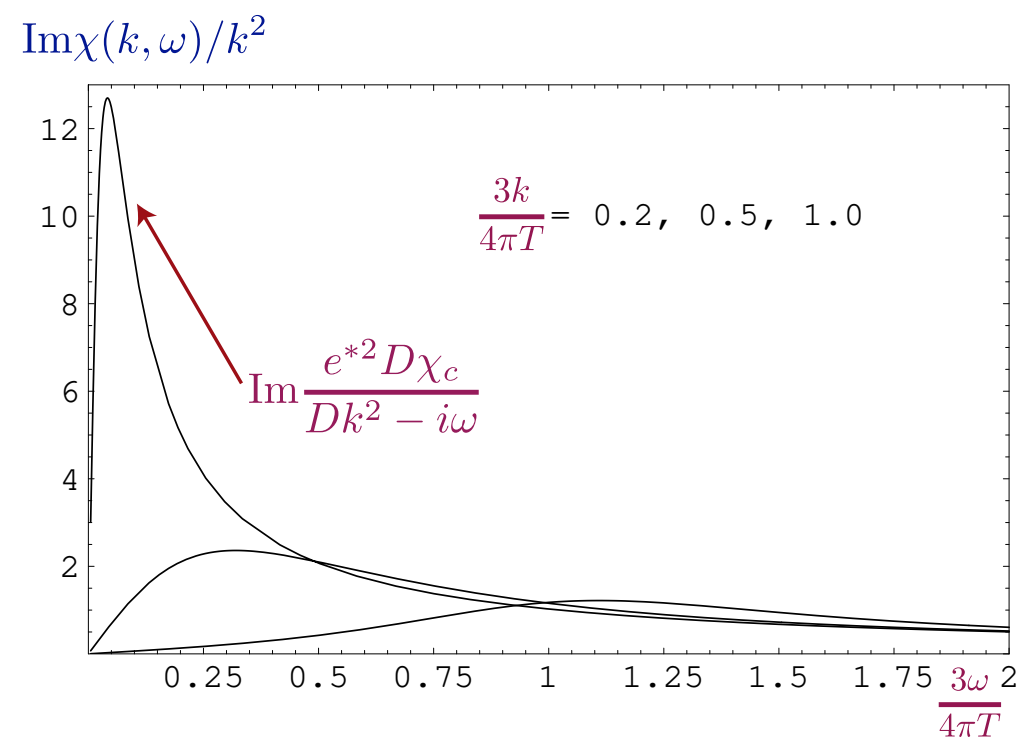

FIG. 9: As in Fig. 8, but for the collision-dominated regime.

defined by

$$
P_{00}^{T}=P_{0 i}^{T}=P_{i 0}^{T}=0 \quad, \quad P_{i j}^{T}=\delta_{i j}-\frac{k_{i} k_{j}}{k^{2}}, \quad P_{\mu \nu}^{L}=\left(\eta_{\mu \nu}-\frac{p_{\mu} p_{\nu}}{p^{2}}\right)-P_{\mu \nu}^{T},
$$

with the indices $i, j$ running over the 2 spatial components. The two functions $K^{T, L}(k, \omega)$ define all the correlators of the density and the current, and the results in Eqs. (3.5) and (3.4) are obtained by taking suitable limits of these functions. We will also need below the general identity

$$
K^{T}(0, \omega)=K^{L}(0, \omega),
$$


which follows from the analyticity of the $T>0$ current correlations at $\mathbf{k}=0$.

The relations of the previous paragraph are completely general and apply to any theory. Specializing to the AdS-Schwarzschild solution of SYM3, the results were found to obey a simple and remarkable identity ${ }^{\underline{43}}$ :

$$
K^{L}(k, \omega) K^{T}(k, \omega)=\mathcal{K}^{2}
$$

where $\mathcal{K}$ is a known pure number, independent of $\omega$ and $k$. It was also shown that such a relation applies to any theory which is equated to classical gravity on $\mathrm{AdS}_{4}$, and is a consequence of the electromagnetic self-duality of its four-dimensional Maxwell sector. The combination of Eqs. (4.3) and (4.4) fully determines the $\chi_{\mu \nu}$ correlators at $\mathbf{k}=0$ : we find $K^{L}(0, \omega)=K^{T}(0, \omega)=\mathcal{K}$, from which it follows that the $\mathbf{k}=0$ conductivity is frequency independent and that $\Phi_{\sigma}=\Theta_{1} \Theta_{2}=K=\mathcal{K}$. These last features are believed to be special to theories which are equivalent to classical gravity, and not hold more generally.

We can obtain further insight into the interpretation of Eq. (4.4) by considering the field theory of the superfluid-insulator transition of lattice bosons at integer filling. As we noted earliear, this is given by the field theory in Eq. (1.3) with the field $\varphi^{a}$ having 2 components. It is known that this 2-component theory of relativistic bosons is equivalent to a dual relativistic theory, $\widetilde{\mathcal{S}}$ of vortices, under the well-known 'particle-vortex' duality $\underline{44}$. Ref. $\underline{43}$ considered the action of this particle-vortex duality on the correlation functions in Eq. (4.1), and found the following interesting relations:

$$
K^{L}(k, \omega) \widetilde{K}^{T}(k, \omega)=1 \quad, \quad K^{T}(k, \omega) \widetilde{K}^{L}(k, \omega)=1
$$

where $\widetilde{K}^{L, T}$ determine the vortex current correlations in $\widetilde{\mathcal{S}}$ as in Eq. (4.1). Unlike Eq. (4.4), Eq. (4.5) does not fully determine the correlation functions at $\mathbf{k}=0$ : it only serves to reduce the 4 unknown functions $K^{L, T}, \widetilde{K}^{L, T}$ to 2 unknown functions. The key property here is that while the theories $\mathcal{S}_{L G}$ and $\widetilde{\mathcal{S}}$ are dual to each other, they are not equivalent, and the theory $\mathcal{S}_{L G}$ is not self-dual.

We now see that Eq. (4.4) implies that the classical gravity theory of SYM3 is self-dual under an analog of particle-vortex duality $\stackrel{43}{\underline{3}}$. It is not expected that this self-duality will hold when quantum gravity corrections are included; equivalently, the SYM3 at finite $N$ is expected to have a frequency dependence in its conductivity at $\mathbf{k}=0$. If we apply the AdS/CFT correspondence to the superfluid-insulator transition, and approximate the latter theory by classical gravity on $\mathrm{AdS}_{4}$, we immediately obtain the self-dual prediction for the conductivity, $\Phi_{\sigma}=1$. This value is not far from that observed in numerous experiments, and we propose here that the AdS/CFT correspondence offers a rationale for understanding such observations. 


\section{HYDRODYNAMIC THEORY}

The successful comparison between the general considerations of Section III, and the exact solution using the AdS/CFT correspondence in Section IV, emboldens us to seek a more general theory of low frequency $\left(\hbar \omega \ll k_{B} T\right)$ transport in the quantum critical regime. We will again present our results for the special case of a relativistic quantum critical point in 2+1 dimensions (a CFT3), but it is clear that similar considerations apply to a wider class of systems. Thus we can envisage applications to the superfluid-insulator transition, and have presented scenarios under which such a framework can be used to interpret measurements of

the Nernst effect in the cuprates $\stackrel{40}{ }$. We have also described a separate set of applications to graphene $\mathrm{e}^{36}$ : while graphene is strictly not a CFT3, the Dirac spectrum of electrons leads to many similar results, especially in the inelastic collision-dominated regime associated with the quantum critical region. These results on graphene are reviewed in a separate paper ${ }^{45}$, where explicit microscopic computations are also discussed.

Our idea is to relax the restricted set of conditions under which the results of Section III were obtained. We will work within the quantum critical regimes of the phase diagrams of Section II but now allow a variety of additional perturbations. First, we will move away from the particle-hole symmetric case, allow a finite density of carriers. For graphene, this means that $\mu$ is no longer pinned at zero; for the antiferromagnets, we can apply an external magnetic field; for the superfluid-insulator transition, the number density need not be commensurate with the underlying lattice. For charged systems, such as the superfluidinsulator transition or graphene, we allow application of an external magnetic field. Finally, we also allow a small density of impurities which can act as a sink of the conserved total momentum of the CFT3. In all cases, the energy scale associated with these perturbations is assumed to be smaller than the dominant energy scale of the quantum critical region, which is $k_{B} T$. The results presented below were obtained in two separate computations, associated with the methods described in Sections III and IV , and are described in the two subsections below.

\section{A. Relativistic magnetohydrodynamics}

With the picture of relaxation to local equilibrium at frequencies $\hbar \omega \ll k_{B} T$ developed in Ref. $\underline{35}$, we postulate that the equations of relativistic magnetohydrodynamics should describe the low frequency transport. The basic principles involved in such a hydrodynamic computation go back to the nineteenth century: conservation of energy, momentum, and charge, and the constraint of the positivity of entropy production. Nevertheless, the required results were not obtained until our recent work ${ }^{40}$ : the general case of a CFT3 in the presence of a chemical potential, magnetic field, and small density of impurities is very intricate, and the guidance provided by the dual gravity formulation was very helpful to us. 
In this approach, we do not have quantitative knowledge of a few transport co-efficients, and this is complementary to our ignorance of the effective couplings in the dual gravity theory to be discussed in Section VB.

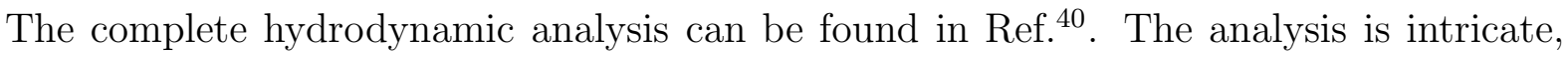
but is mainly a straightforward adaption of the classic procedure outlined by Kadanoff and Martin $\frac{49}{6}$ to the relativistic field theories which describe quantum critical points. We list the steps:

1. Identify the conserved quantities, which are the energy-momentum tensor, $T^{\mu \nu}$, and the particle number current, $J^{\mu}$.

2. Obtain the real time equations of motion, which express the conservation laws:

$$
\partial_{\nu} T^{\mu \nu}=F^{\mu \nu} J_{\nu} \quad, \quad \partial_{\mu} J^{\mu}=0 ;
$$

here $F^{\mu \nu}$ represents the externally applied electric and magnetic fields which can change the net momentum or energy of the system, and we have not written a term describing momentum relaxation by impurities.

3. Identify the state variables which characterize the local thermodynamic state - we choose these to be the density, $\rho$, the temperature $T$, and an average velocity $u^{\mu}$.

4. Express $T^{\mu \nu}$ and $J^{\mu}$ in terms of the state variables and their spatial and temporal gradients; here we use the properties of the observables under a boost by the velocity $u^{\mu}$, and thermodynamic quantities like the energy density, $\varepsilon$, and the pressure, $P$, which are determined from $T$ and $\rho$ by the equation of state of the CFT. We also introduce transport co-efficients associated with the gradient terms.

5. Express the equations of motion in terms of the state variables, and ensure that the entropy production rate is positive $\mathrm{E}^{50}$. This is a key step which ensures relaxation to local equilibrium, and leads to important constraints on the transport co-efficients. In $d=2$, it was found that situations with the velocity $u^{\mu}$ spacetime independent are characterized by only a single independent transport co-efficient $\underline{40}$. This we choose to be the longitudinal conductivity at $B=0$.

6. Solve the initial value problem for the state variables using the linearized equations of motion.

7. Finally, translate this solution to the linear response functions, as described in Ref. $\underline{49}$.

\section{B. Dyonic black hole}

Given the success of the AdS/CFT correspondence for the specific supersymmetric model in Section IV, we boldly assume a similar correspondence for a generic CFT3. We assume 
that each CFT3 is dual to a strongly-coupled theory of gravity on $\mathrm{AdS}_{4}$. Furthermore, given the operators associated with the perturbations away from the pure CFT3 we want to study, we can also deduce the corresponding perturbations away from the dual gravity theory. So far, this correspondence is purely formal and not of much practical use to us. However, we now restrict our attention to the hydrodynamic, collision dominated regime, $\hbar \omega \ll k_{B} T$ of the CFT3. We would like to know the corresponding low energy effective theory describing the quantum gravity theory on $\mathrm{AdS}_{4}$. Here, we make the simplest possible assumption: the effective theory is just the Einstein-Maxwell theory of general relativity and electromagnetism on $\mathrm{AdS}_{4}$. As in Section IV, the temperature $T$ of CFT3 corresponds to introducing a black hole on $\mathrm{AdS}_{4}$ whose Hawking temperature is $T$. The chemical potential, $\mu$, of the CFT3 corresponds to an electric charge on the black hole, and the applied magnetic field maps to a magnetic charge on the black hole. Such a dynoic black hole solution of the Einstein-Maxwell equations is, in fact, known: it is the Reissner-Nordstrom black hole.

We solved the classical Einstein-Maxwell equations for linearized fluctuations about the metric of a dyonic black hole in a space which is asymptotically $\mathrm{AdS}_{4}$. The results were used to obtain correlators of a CFT3 using the prescriptions of the AdS/CFT mapping. As we have noted, we have no detailed knowledge of the strongly-coupled quantum gravity theory which is dual to the CFT3 describing the superfluid-insulator transition in condensed matter systems, or of graphene. Nevertheless, given our postulate that its low energy effective field theory essentially captured by the Einstein-Maxwell theory, we can then obtain a powerful set of results for CFT3s.

\section{Results}

In the end, we obtained complete agreement between the two independent computations in Sections $\mathrm{VA}$ and $\mathrm{VB}$, after allowing for their distinct equations of state. This agreement demonstrates that the assumption of a low energy Einstein-Maxwell effective field theory for a strongly coupled theory of quantum gravity is equivalent to the assumption of hydrodynamic transport for $\hbar \omega \ll k_{B} T$ in a strongly coupled CFT3.

Finally, we turn to our explicit results for quantum critical transport with $\hbar \omega \ll k_{B} T$.

First, consider adding a chemical potential, $\mu$, to the CFT3. This will induce a non-zero number density of carriers $\rho$. The value of $\rho$ is defined so that the total charge density associated with $\rho$ is $e^{*} \rho$. Then the electrical conductivity at a frequency $\omega$ is

$$
\sigma(\omega)=\frac{e^{* 2}}{h} \Phi_{\sigma}+\frac{e^{* 2} \rho^{2} v^{2}}{(\varepsilon+P)} \frac{1}{\left(-i \omega+1 / \tau_{\text {imp }}\right)}
$$

In this section, we are again using the symbol $v$ to denote the characteristic velocity of the CFT3 because we will need $c$ for the physical velocity of light below. Here $\varepsilon$ is the energy density and $P$ is the pressure of the CFT3. We have assumed a small density of impurities 
which lead to a momentum relaxation time $\tau_{\text {imp }} \underline{40} \underline{46}$. In general, $\Phi_{\sigma}, \rho, \varepsilon, P$, and $1 / \tau_{\text {imp }}$ will be functions of $\mu / k_{B} T$ which cannot be computed by hydrodynamic considerations alone. However, apart from $\Phi_{\sigma}$, these quantities are usually amenable to direct perturbative computations in the CFT3, or by quantum Monte Carlo studies. The physical interpretation of Eq. (5.2) should be evident: adding a charge density $\rho$ leads to an additional Drude-like contribution to the conductivity. This extra current cannot be relaxed by collisions between the unequal density of particle and hole excitations, and so requires an impurity relaxation mechanism to yield a finite conductivity in the d.c. limit.

Now consider thermal transport in a CFT3 with a non-zero $\mu$. The d.c. thermal conductivity, $\kappa$, is given by

$$
\kappa=\Phi_{\sigma}\left(\frac{k_{B}^{2} T}{h}\right)\left(\frac{\varepsilon+P}{k_{B} T \rho}\right)^{2},
$$

in the absence of impurity scattering, $1 / \tau_{\text {imp }} \rightarrow 0$. This is a Wiedemann-Franz-like relation, connecting the thermal conductivity to the electrical conductivity in the $\mu=0 \mathrm{CFT}$. Note that $\kappa$ diverges as $\rho \rightarrow 0$, and so the thermal conductivity of the $\mu=0$ CFT is infinite.

Next, turn on a small magnetic field $B$; we assume that $B$ is small enough that the spacing between the Landau levels is not as large as $k_{B} T$. The case of large Landau level spacing is also experimentally important, but cannot be addressed by the present analysis. Initially, consider the case $\mu=0$. In this case, the result Eq. (5.3) for the thermal conductivity is replaced by

$$
\kappa=\frac{1}{\Phi_{\sigma}}\left(\frac{k_{B}^{2} T}{h}\right)\left(\frac{\varepsilon+P}{k_{B} T B /\left(h c / e^{*}\right)}\right)^{2}
$$

also in the absence of impurity scattering, $1 / \tau_{\text {imp }} \rightarrow 0$. This result relates $\kappa$ to the electrical resistance at criticality, and so can be viewed as Wiedemann-Franz-like relation for the vortices. A similar $1 / B^{2}$ dependence of $\kappa$ appeared in the Boltzmann equation analysis of Ref. $\stackrel{47}{ }$, but our more general analysis applies in a wider and distinct regime ${ }^{36}$, and relates the co-efficient to other observables.

We have obtained a full set of results for the frequency-dependent thermo-electric response functions at non-zero $B$ and $\mu$. The results are lengthy and we refer the reader to Ref. $\underline{\underline{40}}$ for explicit expressions. Here we only note that the characteristic feature $\underline{40}+48$ of these results is a new hydrodynamic cyclotron resonance. The usual cyclotron resonance occurs at the classical cyclotron frequency, which is independent of the particle density and temperature; further, in a Galilean-invariant system this resonance is not broadened by electron-electron interactions alone, and requires impurities for non-zero damping. The situtation for our hydrodynamic resonance is very different. It occurs in a collision-dominated regime, and its frequency depends on the density and temperature: the explicit expression for the resonance frequency is

$$
\omega_{c}=\frac{e^{*} B \rho v^{2}}{c(\varepsilon+P)} .
$$


Further, the cyclotron resonance involves particle and hole excitations moving in opposite directions, and collisions between them can damp the resonance even in the absence of impurities. Our expression for this intrinsic damping frequency is $\underline{40}, \underline{48}$

$$
\gamma=\frac{e^{* 2}}{h} \Phi_{\sigma} \frac{B^{2} v^{2}}{c^{2}(\varepsilon+P)},
$$

relating it to the quantum-critical conductivity as a measure of collisions between counterpropagating particles and holes. We refer the reader to a separate discussion $\underline{36}$ of the experimental conditions under which this hydrodynamic cyclotron resonance may be observed.

\section{THE CUPRATE SUPERCONDUCTORS}

We close this article by mentioning application to the cuprate superconductors. The phenomenology of these materials is very involved, and they have so far resisted a global interpretation in terms of the canonical quantum-critical crossover phase diagrams discussed here. Surely, one of the important complexities is that they involve at least two order parameters: those associated with $d$-wave superconductivity and with SDWs (both commensurate and incommensurate with the underlying lattice). In addition there are also topological changes in the Fermi surface discussed in Section ID and Fig. 4.

Here we mention recent ideas $51-54$ which attempt a synthesis based upon a combination of the crossover phase diagrams we have discussed here. The ideas build upon the results of a number of recent experiments $\underline{\underline{55}} \underline{\underline{59}}$ which have explored the phase diagram of both the hole- and electron-doped cuprates in a magnetic field applied perpendicular to the layers. We do not wish to enter into the intricate details of the structure of the phase diagram in the field-doping plane, and refer the reader to recent reviews $s^{\underline{53}} \underline{\underline{54}}$. We will limit ourselves to the assertion that the experiments support the proposa $\left.\right|^{58}$ that the underlying metallic state of the cuprates undergoes an SDW transition as in Fig. 7 along with the topological change of the Fermi surface, which was illustrated in Fig. 4 for the simplest case of commensurate ordering. Recent computations $\underline{\underline{60}}$ and observations ${ }^{61}$ of thermoelectric effects have provided additional support for this proposal.

The main ingredient missing from Fig. 7 is $d$-wave superconductivity. It is known that the SDW fluctuations can induce $d$-wave pairing for the Fermi surface configuration shown in Fig. 4. So let us superimpose a superconducting region on Fig. 7, and this leads us to Fig. 10, Arguments that this superconducting region will have the dome shape around the quantum critical point were made in Ref. $\frac{52}{.}$. Here we wish to draw attention to a crucial new feature shown in Fig. 10. The onset of superconductivity shifts the spin ordering quantum critical point from its apparent position in the underlying metal at $x=x_{m}$, to its actual position in the superconductor at $x=x_{s}$. This shift is indicated by the large arrow in Fig. 10, We have

argued ${ }^{52}-\underline{54}$ that this shift helps resolve a number of long-standing puzzles in understanding 


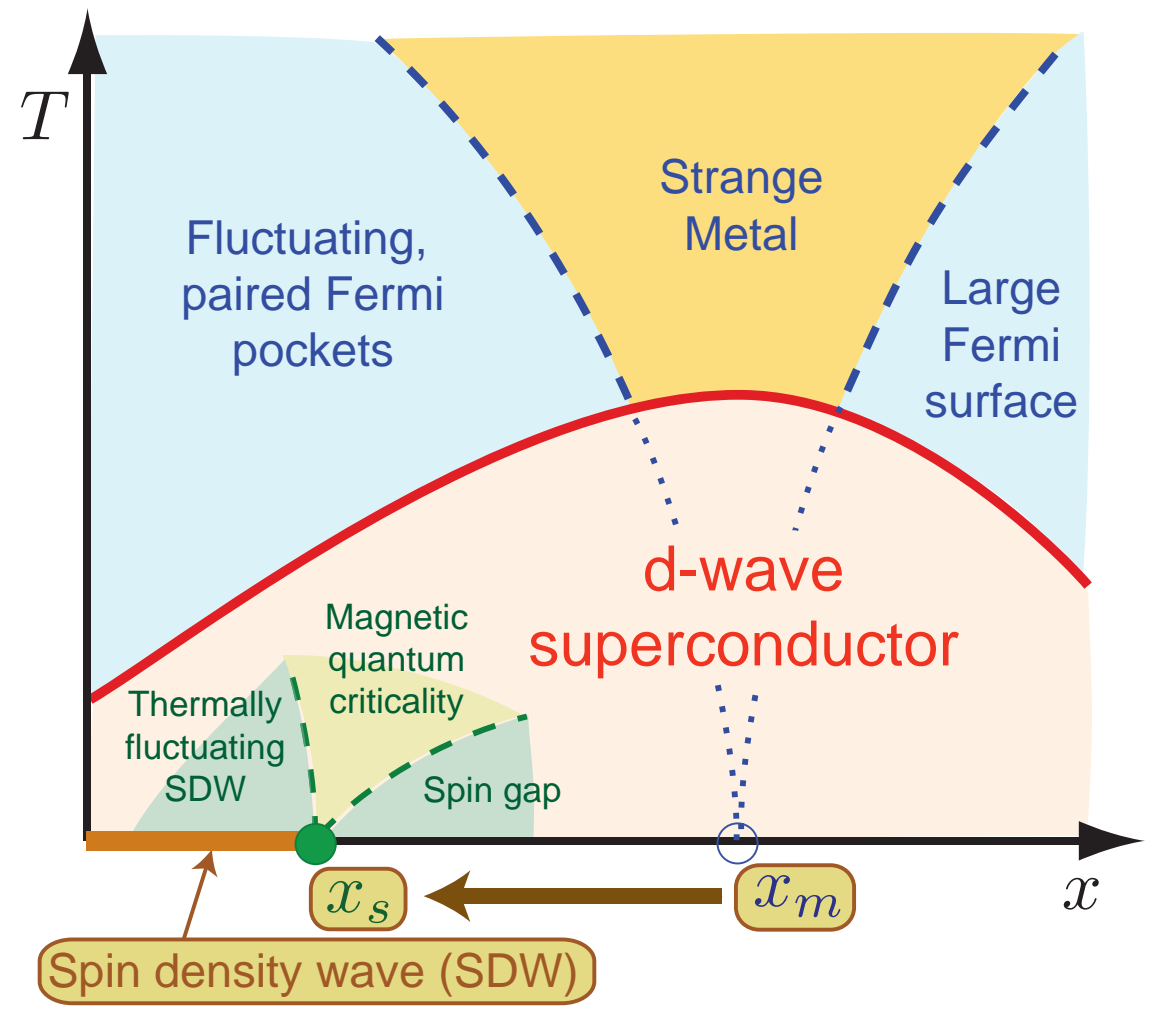

FIG. 10: Influence of the onset of superconductivity on the crossovers near the SDW ordering transition in Fig. [7]

the experimental observations in the cuprates in terms of the quantum-critical crossover phase diagrams.

What is the physical origin of the shift in the position of the quantum critical point? In phenomenological terms, this shift can be understood as a competition between the SDW and superconducting order parameters ${ }^{62}$. Once there is an onset of superconductivity, it repels the incipient SDW ordering, and shifts it away from the superconducting region to lower carrier concentration. A microscopic theory has also been presented in Ref $\sqrt[52]{ }$ : it requires special attention to the physics of the Fermi surface, and the fact that SDW ordering and superconductivity are 'eating up' the same portions of the Fermi surface. Such phenomena are especially pronounced near the Mott insulating state at zero doping.

Once the shift has occurred, the crossovers near the spin-ordering transition within the superconductor are especially simple. There are no longer any Fermi surfaces to contend with, and so we can largely focus on the fluctuations of the order parameter. The latter reduce $^{63}$ to those presented in Fig. 5 , with critical theories like those presented in Sections $\underline{\mathrm{IA}}$ and IB.

So the bottom line is that the proposed cuprate phase diagram in Fig. 10 is a combination of the crossovers in Fig. 5 and 7 , along with the all-important shift in the position of the quantum critical point. 
It is now interesting to examine the subtle evolution in the physics as the temperature is lowered for $x_{s}<x<x_{m}$. Above $T_{c}$, we have a metallic state with fluctuating SDW order whose strength increases as $T$ is lowered. Once we cross $T_{c}$, the growth in SDW ordering is arrested (because of the competition with superconductivity); the system is now to the right of the SDW ordering quantum critical point, and starts to recover aspects of the physics of the large Fermi surface. However, it has been argued $\underline{\underline{63}}$ that forms of translational symmetry breaking, such as VBS or nematic ordering may appear as $T \rightarrow 0$ for $x_{s}<x<x_{m}$. In this case, the left crossover line emerging from $x_{m}$ in Fig. 10 would become an actual thermal phase transition at which lattice symmetry is restored.

\section{Acknowledgments}

This research was supported by the National Science Foundation under grant DMR0757145), by the FQXi foundation, and by a MURI grant from AFOSR.

1 S. Sachdev, Quantum Phase Transitions. Cambridge University Press, Cambridge U.K. (1999).

2 S. Sachdev, Nature Physics 4, 173 (2008); S. Sachdev and M. Müller, Journal of Physics: Condensed Matter 21, 164216 (2009).

3 M. Troyer, M. Imada, and K. Ueda, J. Phys. Soc. Japan 66, 2957 (1997).

4 M. Matsumoto, C. Yasuda, S. Todo, and H. Takayama, Phys. Rev.B 65, 014407 (2002).

5 S. Wenzel and W. Janke, Phys. Rev. B 79, 014410 (2009).

6 M. Campostrini et al., Phys. Rev. B 65, 144520 (2002).

7 A. W. Sandvik and D. J. Scalapino, Phys. Rev. Lett. 72, 2777 (1994).

8 Y. Matsushita, M. P. Gelfand, and C. Ishii, J. Phys. Soc. Jpn 66, 3648 (1997).

9 A. F. Albuquerque, M. Troyer, and J. Oitmaa, Phys. Rev. B 78, 132402 (2008).

10 Ch. Ruegg et al., Phys. Rev. Lett. 100, 205701 (2008).

11 B. Normand and T. M. Rice, Phys. Rev. B 56, 8760 (1997).

12 S. Sachdev, Phys. Rev. B 55, 142 (1997).

13 N. Read and S. Sachdev, Phys. Rev. Lett. 62, 1694 (1989).

14 M. Tamura, A. Nakao and R. Kato, J. Phys. Soc. Japan 75, 093701 (2006).

15 Y. Shimizu, H. Akimoto, H. Tsujii, A. Tajima, and R. Kato, Phys. Rev. Lett. 99, 256403 (2007).

16 T. Senthil, A. Vishwanath, L. Balents, S. Sachdev, and M. P. A. Fisher, Science 303, 1490 (2004).

17 A. W. Sandvik, Phys. Rev. Lett. 98, 227202 (2007).

18 J. Lou, A. W. Sandvik, and N Kawashima, arXiv:0908.0740

19 R. G. Melko and R. K. Kaul, Phys. Rev. Lett. 100, 017203 (2008); Phys. Rev. B 78, 014417 (2008). 
20 F.-J. Jiang, M. Nyfeler, S. Chandrasekharan, and U.-J. Wiese, Stat. Mech. (2008) P02009.

21 A. B. Kuklov, M. Matsumoto, N. V. Prokof'ev, B. V. Svistunov, and M. Troyer, Phys. Rev. Lett. 101, 050405 (2008).

22 A. H. Castro Neto, F. Guinea, N. M. Peres, K. S. Novoselov, and A. K. Geim, Rev. Mod. Phys. 81, 109 (2009).

23 N. P. Armitage et al., Phys. Rev. Lett. 88, 257001 (2002).

24 T. Helm et al., arXiv:0906.1431

25 S. Sachdev, A. V. Chubukov, and A. Sokol, Phys. Rev. B 51, 14874 (1995).

26 M. T. Béal-Monod and K. Maki, Phys. Rev. Lett. 34, 1461 (1975).

27 J. A. Hertz, Phys. Rev. B 14, 1165 (1976).

28 Ar. Abanov and A. Chubukov, Phys. Rev. Lett. 93, 255702 (2004).

29 S. Chakravarty, B. I. Halperin, and D. R. Nelson, Phys. Rev. B 39, 2344 (1989).

30 S. Sachdev and J. Ye, Phys. Rev. Lett. 69, 2411 (1992); A. V. Chubukov, S. Sachdev, and J. Ye, Phys. Rev. B 49, 11919 (1994).

31 D. E. Sheehy and J. Schmalian, Phys. Rev. Lett. 99, 226803 (2007).

32 A. J. Millis, Phys. Rev. B 48, 7183 (1993).

33 H. v. Löhneysen, A. Rosch, M. Vojta, and P. Wölfle, Rev. Mod. Phys. 79, 1015 (2007).

34 M. P. A. Fisher, P. B. Weichman, G. Grinstein, and D. S. Fisher, Phys. Rev. B 40: 546, 1989.

35 K. Damle and S. Sachdev, Phys. Rev. B 56, 8714 (1997).

36 L. Fritz, J. Schmalian, M. Müller and S. Sachdev, Phys. Rev. B 78, 085416 (2008); M. Müller and S. Sachdev, Phys. Rev. B 78, 115419 (2008); M. Müller, L. Fritz, and S. Sachdev, Phys. Rev. B 78, 115406 (2008).

37 For the case of neutral boson superfluids (but not charged systems like graphene), hydrodynamic 'long-time tails' cause the constants $D$ and $\Theta_{2}$ to acquire a weak logarithmic dependence on $\hbar \omega / k_{B} T$ at small $\omega$ in a sample with perfect momentum conservation. P. Kovtun and L. G. Yaffe, Phys. Rev. D 68, 025007 (2003).

38 P. K. Kovtun, D. T. Son, and A. Starinets, Phys. Rev. Lett. 94, 11601 (2005).

39 M. P. A. Fisher, G. Grinstein, and S. M. Girvin, Phys. Rev. Lett. 64, 587 (1990); M. P. A. Fisher, Phys. Rev. Lett. 65, 923 (1990).

40 S. A. Hartnoll, P. K. Kovtun, M. Müller, and S. Sachdev, Phys. Rev. B 76, 144502 (2007).

41 M. Müller, J. Schmalian, and L. Fritz, Phys. Rev. Lett. 103, 025301 (2009).

42 N. Itzhaki, J. M. Maldacena, J. Sonnenschein and S. Yankielowicz, Phys. Rev. D 58, 046004 (1998).

43 C. P. Herzog, P. Kovtun. S. Sachdev, and D. T. Son, Phys. Rev. D 75, 085020 (2007).

44 C. Dasgupta and B. I. Halperin, Phys. Rev. Lett. 47, 1556 (1981).

45 M. Müller, L. Fritz, S. Sachdev, and J. Schmalian, Advances in Theoretical Physics: Landau Memorial Conference, Chernogolovka 2008, AIP Conference Proceedings 1134, 170 (2009).

46 S. A. Hartnoll and C. P. Herzog, Phys. Rev. D 77, 106009 (2008). 
47 M. J. Bhaseen, A. G. Green, and S. L. Sondhi, Phys. Rev. Lett. 98, 166801 (2007); Phys. Rev. B 79, 094502 (2009).

48 S. A. Hartnoll and C. P. Herzog, Phys. Rev. D 76, 106012 (2007).

49 L. P. Kadanoff and P. C. Martin, Annals of Physics 24, 419-469 (1963).

50 Landau, L. D. \& Lifshitz, E. M. Fluid Mechanics, Section 127 (Butterworth-Heinemann, Oxford, 1987).

51 V. Galitski and S. Sachdev, Phys. Rev. B 79, 134512 (2009).

52 E. G. Moon and S. Sachdev, Phys. Rev. B 80, 035117 (2009).

53 S. Sachdev, arXiv:0907.0008.

54 S. Sachdev, arXiv:0910.0846.

55 N. Doiron-Leyraud et al., Nature 447, 565 (2007).

56 D. LeBoeuf et al., Nature 450, 533 (2007).

57 S. E. Sebastian et al., Nature 454, 200 (2008).

58 R. Daou et al., Nature Physics 5, 31 (2009).

59 T. Helm et al., arXiv:0906.1431.

60 A. Hackl, M. Vojta and S. Sachdev, arXiv:0908.1088.

61 J. Chang et al., arXiv:0907.5039.

62 E. Demler, S. Sachdev and Y. Zhang, Phys. Rev. Lett. 87, 067202 (2001); Y. Zhang, E. Demler and S. Sachdev, Phys. Rev. B 66, 094501 (2002).

63 R. K. Kaul, M. A. Metlitski, S. Sachdev and C. Xu, Phys. Rev. B 78, 045110 (2008). 